\title{
Diesel Exhaust Particles Induce Local IgE Production In Vivo and Alter the Pattern of IgE Messenger RNA Isoforms
}

David Diaz-Sanchez, Anton Robert Dotson, Hiroshi Takenaka, and Andrew Saxon

The Hart and Louise Lyon Laboratory; Division of Clinical Immunology/Allergy, Department of Medicine, UCLA School of Medicine, University of California, Los Angeles, California 90024-1680

\begin{abstract}
Diesel exhaust particles (DEP) have been implicated in the increased incidence of allergic airway disorders. We investigated the effects of DEP on localized immunoglobulin production by performing nasal challenges with varying doses of DEP and analyzing the local immune response in nasal lavages obtained before and after. A significant rise in nasal IgE but not IgG, IgA, IgM, or albumin was observed in subjects $4 \mathrm{~d}$ after challenge with $0.30 \mathrm{mg}$ DEP, equivalent to exposure on an average Los Angeles day. Direct evidence for DEP-enhanced local production of IgE was that challenge increased the number of IgE-secreting cells in lavage fluid from $<1$ in $2,000,000$ to $>1$ in 100,000 but did not alter the number of IgA-secreting cells. There was a concomitant increase in epsilon mRNA production in the lavage cells. Additionally, DEP altered the relative amounts of five different epsilon mRNAs generated by alternative splicing, mRNAs that code for different IgE proteins. These results show that DEP exposure in vivo causes both quantitative and qualitative changes in local IgE production. The implication is that natural exposure to DEP may result in increased expression of respiratory allergic disease. ( $J$. Clin. Invest. 1994. 94:1417-1425.) Key words: IgE, human • pollution - allergic antibody response - mucosal immunity • allergy
\end{abstract}

\section{Introduction}

Many epidemiological studies have suggested a link between air quality, the increase of atmospheric pollutants, and the in crease in the incidence and morbidity of allergic airway disease (1-5). The hallmark of these diseases is an increase in the production of IgE. An example is the increase in allergic airway disease among the Japanese which has coincided with the industrialization of Japan since the 1940s. From a rare disease before 1950 , allergic airway disease has evolved into a significant cause of mortality. In this time there has been a 500 -fold in-

Address correspondence to David Diaz-Sanchez, Ph.D., Division of Clinical Immunology/Allergy, Department of Medicine, 52-175 Center for Health Sciences, UCLA School of Medicine, Los Angeles, CA 90024-1680. Dr. Hiroshi Takenaka's present address is The Department of Otorhinolaryngology, Kyoto Prefectural University of Medicine, Kawaramach, Hirokoji, Kamigyoku, Kyoto 602 Japan.

Received for publication 17 March 1994 and in revised form 24 June 1994

J. Clin. Invest.

(C) The American Society for Clinical Investigation, Inc. 0021-9738/94/10/1417/09 \$2.00

Volume 94, October 1994, 1417-1425 crease in the number of diesel cars and a $>1,000$-fold increase in the amount of polyaromatic hydrocarbons (PAH) ${ }^{1}$ in diesel exhaust particles (DEP) (5). Many of these PAH are also present in tobacco smoke (6), which is also associated with increased IgE levels. A great deal of attention has been directed to the potential immunosuppressive and carcinogenic properties of such PAH. In this study, we have addressed directly in vivo effects of DEP on humans as related to allergic responses.

Miyamoto and colleagues (7-9) performed both in vivo and in vitro studies with mice and demonstrated a direct effect of DEP on IgE antibody production. Simultaneous administration of DEP increased IgE production to bystander antigens such as ovalbumin and Japanese cedar pollen when injected intraperitoneally $(7,9)$. In addition, intranasal administration of DEP caused an increase in the IgE but not the IgG response $(8,9)$. Previous reports have demonstrated that some PAH can have direct effects on lymphocytes, both $T$ cells $(10)$ and on B cells (11). Our laboratory has shown previously that PAH extracted from DEP could directly affect human B cells and enhance IgE production in vitro by modifying ongoing transcriptional programs $(11 a)$. In addition, PAH from DEP dramatically altered the pattern of mRNAs coding for distinct isoforms of the epsilon chain by upregulating production of some isoforms to a much greater extent than others $(11 a)$. Here, we extend these studies to examine the effects of DEP in vivo in humans. In this study, we demonstrate for the first time that DEP can specifically enhance in vivo IgE production at the human upper respiratory mucosa. This increase is due to a local increase in the number of IgE-secreting cells. Furthermore, we show that we can detect different isoforms of epsilon mRNA in cells obtained directly from nasal washes and that the epsilon mRNA isoform pattern observed is altered after provocation with DEP.

\section{Methods}

Subjects. 11 healthy, nonsmoking volunteers (6 males and 5 females) with ages ranging from 23 to $48 \mathrm{yr}$ old were recruited for this study. Seven had no history of asthma, allergic rhinitis, or any allergic or respiratory illness, while four had a history of mild seasonal allergies but were asymptomatic and had not taken any medication $3 \mathrm{~d}$ before or at any time during the duration of the study. All atopics were outside their allergic season. None of the volunteers had previously had any known extensive exposure to pollutants.

Nasal washes and DEP provocation. The subjects sat and closed their nasopharynx while tilting their necks back $45^{\circ}$ from the horizontal. Into each nostril $5 \mathrm{ml}$ of normal saline that had been prewarmed to $37^{\circ} \mathrm{C}$ was delivered by a pipette. After $10 \mathrm{~s}$, during which the subjects shook their heads softly from side to side, they brought their heads

1. Abbreviations used in this paper: DEP, diesel exhaust particles; PAH, polyaromatic hydrocarbons. 
forward, expelling the wash fluid into a plastic receptacle, and the fluid was then transferred to a separate tube. The initial wash was discarded since we observed that this first wash had elevated levels of albumin compared with subsequent washes. The subjects then performed four subsequent nasal washes at 10-min intervals, with each wash collected into a separate tube. The tubes were centrifuged at $350 \mathrm{~g}$ for $10 \mathrm{~min}$ at $4^{\circ} \mathrm{C}$. The aqueous supernatants were then removed and stored at $-70^{\circ} \mathrm{C}$ until needed. When cells were needed for filter spot-ELISA or for RNA extraction, the number of nasal washes was increased to eight. The number of cells recovered remained reasonably constant for each wash, but the percentage of epithelial cells decreased with repeated washing, resulting in an increase in mononuclear cell numbers. $10 \mathrm{~min}$ after the last wash, either $1,0.3,0.15$, or $0 \mathrm{mg}$ of DEP suspended in $200 \mu \mathrm{l}$ of saline was sprayed into each nostril. All subjects were exposed to all four doses of DEP. Each challenge was single-blinded, and there was a duration of at least $18 \mathrm{~d}$ between exposures. In each case on each day, the first wash was discarded.

DEP were obtained by collecting the exhaust from a light duty diesel passenger car onto a cyclone impactor equipped with a dilution tunnel constant volume sampler system as described and were the kind gift of Dr. Shigeru Takafuji (Department of Medicine and Physical Therapy, Faculty of Medicine, University of Tokyo, Tokyo, Japan). These particles were collected, processed, and preserved as described previously (7). Nasal washes on the subjects were performed 4, 7, and $10 \mathrm{~d}$ after the nasal provocation at day 0 . Each set of washes was performed at the same time of day to avoid any diurnal variation. All studies had been approved by the Human Protection of Subjects Committee of the University of California at Los Angeles.

Immunoglobulin and albumin determination. The levels of the Ig isotypes (IgE, IgG, IgG4, IgG3, IgA, and IgM) in the supernatants of cultured cells were measured by isotype-specific ELISAs as described previously with minor modifications $(12,13)$. For the determination of albumin levels, an identical ELISA procedure was used. The antibodies used for this assay were anti-human albumin mAb (Sigma Chemical Co., St. Louis, MO) at $7.5 \mu \mathrm{g} / \mathrm{ml}$ to coat the microtiter plates (Nunc, Roskilde, Denmark) and alkaline phosphatase-labeled antialbumin (The Binding Site, Birmingham, United Kingdom) at 1:3000 for detection. All samples were run in duplicate and repeated if there was more than a $5 \%$ variation between the duplicates.

Quantitation of IgE- and IgA-secreting cells. To detect and enumerate IgE- and IgA-secreting B cells, a previously described $(14,15)$ filter spot-ELISA was used with minor modifications. Briefly, nitrocellulosebottomed 96-well Milliliter HA plates (Millipore Corp., Bedford, MA) were coated overnight at $4^{\circ} \mathrm{C}$ with either $2 \mu \mathrm{g} / \mathrm{ml}$ of anti-human IgE mAb CIA-E-7.12 and CIA-E-4.15 (12) or affinity-purified rabbit antihuman IgA (Tago, Inc., Burlingame, CA). The plates were then washed in PBS $/ 0.05 \%$ Tween 20 and blocked for $1 \mathrm{~h}$ with $0.1 \%$ gelatin. The plates were washed, and known numbers of unfractionated cells recovered from the nasal washes were added into each well in $0.1 \mathrm{ml}$ of RPMI with $10 \%$ FCS. The sensitivity of the IgE spot assay was determined by using serial dilutions of IgE-producing cells 2C4/F3 (16), direct counts of these cells were compared with the number of spots detected and used to construct a standard curve. The assay was shown to be specific because no plaques could be detected when using myeloma cells producing other immunoglobulin isotypes. Similarly, IgA-producing myeloma cells (GM1056) (17) were used to construct a standard curve for the IgA filter spot assay. Incubation was overnight at $37^{\circ} \mathrm{C}$ in a humidified $\mathrm{CO}_{2}$ incubator. After extensive washing, $100 \mu \mathrm{l} /$ well of alkaline phosphate-labeled antibodies reactive with the $\mathrm{Fc}$ portion of the corresponding Ig was added and incubated for $2 \mathrm{~h}$ at room temperature (IgE: 1:3,000 [Kirkegaard and Perry Laboratories, Inc., Gaithersburg, MD]; IgA: 1:3000 [Tago, Inc.]). The plates were incubated at $37^{\circ} \mathrm{C}$ for $2 \mathrm{~h}$ and washed six times before development with BCIP/NBT substrate solution. The plates were dried, the blue spots were enumerated, and the number of Ig-secreting cells was calculated by reading off the appropriate standard curve. Similar to previous reports, positive reactions were recognized as circular granulated foci with diameters $>0.05 \mathrm{~mm}$ (14). Samples were run in quintuplicate and serially diluted.

Reverse transcription-polymerase chain reaction. Alternate splicing of the single human functional epsilon heavy chain gene results in at least five different mRNA isoforms (18). We developed modified polymerase chain reaction techniques to compare and quantitate the amounts of the different isoforms of epsilon mRNA produced. Total cellular RNA from the cells recovered from nasal washes $\left(1-5 \times 10^{6}\right.$ cells/sample) was isolated and then reverse transcribed using Moloney murine leukemia virus reverse transcriptase (Bethesda Research Laboratories, Gaithersburg, MD) as described previously (19).

To lower nonspecific background bands, all reactions were performed in laminar flow hoods with pipettes dedicated for PCR use. The PCR strategies were based on that reported by Go et al. (20). Each one of the downstream primers used in each PCR reaction was kinased with [ $\left.\gamma^{32} \mathrm{P}\right]$ ATP. As $1 \mu \mathrm{g}$ of the oligonucleotide was labeled and brought up to $100 \mu \mathrm{l}$ before the G-25 spin, it was assumed that the labeled oligonucleotide concentration was $10 \mathrm{ng} / \mu \mathrm{l}$. The reaction mixtures and cycle times were chosen after extensive titration of each reagent and time to ensure equal amplification of all products.

Two different PCR reactions were performed. The position in the epsilon gene of each set of primer used is shown in the top of Fig. 1. The first PCR, termed PCR1', was used to amplify the three epsilon mRNA isoforms CH4-M1 '-M2, CH4-M2', and CH4-M2" (18) and the housekeeping gene $\beta$-actin. It used a supermix reaction mixture that contained per sample: $1 \mathrm{U}$ Perfect Match (Stratagene, La Jolla, CA) $10 \%$ DMSO, $50 \mathrm{mM} \mathrm{KCl}, 20 \mathrm{mM}$ Tris- $\mathrm{HCl}$ (pH 8.4), $2.1 \mathrm{mM} \mathrm{MgCl}$, $0.1 \%$ (wt/vol) gelatin, $0.2 \mathrm{mM}$ dNTP, $2.0 \mathrm{U}$ Taq polymerase (Promega Corp., Madison, WI), $30 \mathrm{pmol}$ of the upstream oligonucleotide primer for epsilon isoforms, 25 pmol upstream primers for $\beta$-actin, and $30 \mathrm{pmol}$ $\left(2 \times 10^{6} \mathrm{cpm}\right)$ of each of the downstream primers which previously had been ${ }^{32} \mathrm{P}$-end-labeled. Aliquots were then amplified by PCR on a preheated $\left(94^{\circ} \mathrm{C}\right)$ thermal cycler (Perkin-Elmer Corp., Norwalk, CT) for 25 cycles. Each cycle consisted of denaturation at $94^{\circ} \mathrm{C}$ for $1 \mathrm{~min}$, followed by $1 \mathrm{~min}$ at $67^{\circ} \mathrm{C}$, and then $1 \mathrm{~min} 30 \mathrm{~s}$ at $72^{\circ} \mathrm{C}$. The primers used for PCR1' were (Fig. 1): A, 5'-GGCCATCGATAGGCAGCGAGCCCCTTCACAGACCG-3' and B, 5'-GGCCGTCGACGACGGGGATGCCAGGCAGTTGGATC-3' to amplify the epsilon isoforms CH4-M1 '-M2, CH4-M2', and CH4-M2" (18); and 5'-GTGGGGCGCCCCAGGCACCA-3' and 5'-CTCCTTAATGTCACGCACGATTTC$3^{\prime}$ to amplify $\beta$-actin (21).

The second PCR, termed PCR2', was used to amplify two other epsilon mRNA isoforms, CH4-S (the classic secreted isoform) and the alternative spliced $\mathrm{CH} 4{ }^{\prime}-\mathrm{CH} 5$ forms. The reaction mixture used was identical as for PCR1' except that the $\mathrm{MgCl}_{2}$ concentration was increased to $2.4 \mathrm{mM}$. The reaction was carried out with denaturation at $94^{\circ} \mathrm{C}$ for $1 \mathrm{~min}$, followed by $1 \mathrm{~min}$, followed by $1 \mathrm{~min} 30 \mathrm{~s}$ at $65^{\circ} \mathrm{C}$, and then $1 \mathrm{~min} 30 \mathrm{~s}$ at $72^{\circ} \mathrm{C}$. The primers used for PCR2' were: $\mathrm{C}, 5^{\prime}$ TCATGCGGTCCACGACCAAGA-3' and D, 5'-GTCATTGCAACAGTGGACAG-3' to amplify the epsilon isoforms (18); and 5'-CGCGACAAGATGACCCAGATC-3' and ' 5'-ATCACGATGCCAGTGGTACGG-3' to amplify $\beta$-actin (22). A different primer pair was used for $\beta$-actin for this reaction as the ones used in PCR1' resulted in an amplified fragment that could not be resolved from the other fragments.

For quantitation, aliquots of $20 \mu \mathrm{l}$ of each PCR product were run on an $8 \%$ nondenaturing polyacrylamide gel at $100 \mathrm{~V}$ for $5 \mathrm{~h}$, dried on a gel drier (model 543; Bio-Rad Laboratories, Hercules, CA) for 45 $\mathrm{min}$ at $65^{\circ} \mathrm{C}$ and then exposed to Kodak XAR-5 film for 8-12 h. In place of a densitometer, the autoradiograph was scanned on an Apple One 24-bit scanner at 300 dots per inch and converted into a TIFF uncompressed format, and the resultant pattern was analyzed using the Scan Analysis program from Biosoft (Cambridge, United Kingdom) which delineates peaks and integrates the area under each peak.

Validation of the PCR strategies and quantitation. Plasmids were constructed using the Bluescript (Stratagene) vector as described previously (19). Each contained cDNA for one of the epsilon isoforms or the $\beta$-actin. PCR titration of individual plasmids showed that for each this PCR strategy was sensitive to $10^{2}$ copies of template. Plasmids containing cDNA for CH4-M1 '-M2, CH4-M2', CH4-M2", and $\beta$-actin were then added together in 10 -fold serial dilutions and subjected to PCR1'. There was a $\log$-linear correlation between the number of starting copies of the plasmid and the PCR yield as quantified by densitome- 


\section{Epsilon mRNAs and Proteins Generated by 3' Splicing}
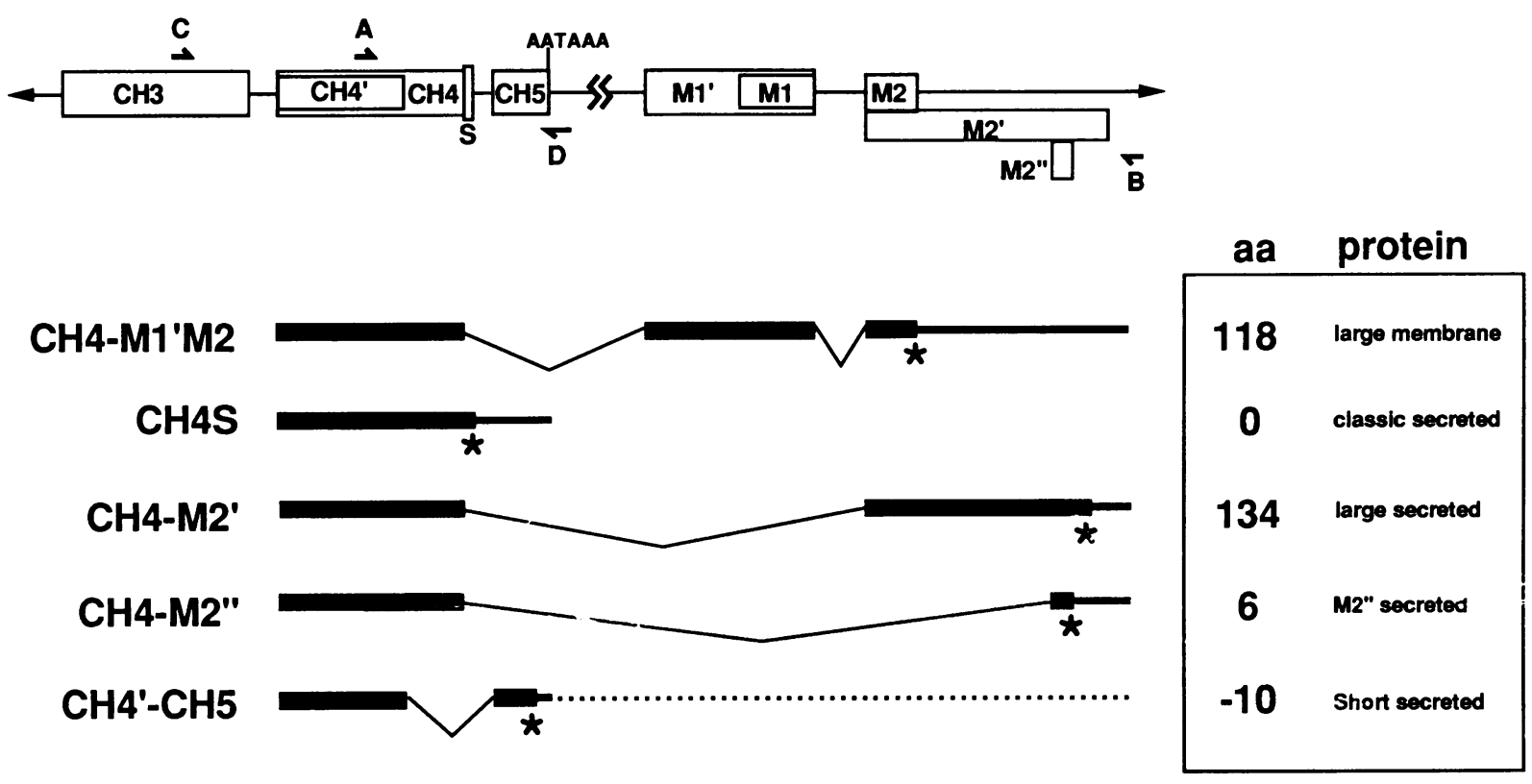

Figure 1. Diagram of epsilon mRNA isoforms and proteins generated by alternative 3 ' splicing. The upper part of this figure shows a schematic representation of the $3^{\prime}$ end of the human epsilon gene with alternative exon sequences shown as boxes (18). The positions of the primer pairs used for PCR1' $(A$ and $B$ ) and PCR2' $(C$ and $D$ ) to measure the amounts of the various epsilon mRNAs are shown. In the lower part of the figure, the $3^{\prime}$ ends of the five epsilon mRNA species analyzed in our two PCR amplifications are shown. The bold horizontal bars represent translated exon sequence. The asterisks represent the positions of stop codons, and the thinner horizontal lines represent $3^{\prime}$ untranslated sequence. On the right hand side, the resulting epsilon protein is shown, and its size in amino acids relative to the size of classic secreted epsilon protein is given.

try for each cDNA. Importantly, a log-linear correlation was still observed for each cDNA, and a reduction of the intensity of the amplified products was not observed. The individual rates of amplification were determined by the slope of the line, an increase in the starting concentration of cDNA of one isoform did not alter the amplification rates of the other isoforms. The PCR product increased according to its amplification rate and the number of starting plasmid, even when this was $10^{10}$ copies. When plasmids containing $\mathrm{cDNA}$ for $\mathrm{CH} 4-\mathrm{S}$ and $\mathrm{CH} 4^{\prime}-\mathrm{CH} 5$ and $\beta$-actin were added and subjected to PCR2', similar findings were observed. In both PCR procedures, the amplification rates for each isoform were similar but not identical, therefore, for each isoform a correction factor was calculated to standardize the rates. The individual isoform amplification rates were thus normalized to that of $\beta$-actin mRNA. Reproducible amplification rates were obtained for each isoform, after PCR. $\beta$ Actin is a housekeeping gene constitutively expressed by cells and is a direct measure of the amount of cells from which RNA was extracted. Therefore, as all samples were amplified with primers for $\beta$-actin, all densitometry values for epsilon isoforms were normalized to $\beta$-actin expression. All samples were run in duplicate and repeated if there was more than a $5 \%$ variation between the duplicates.

To confirm that the amplification rates were identical in both plasmids and cells, the increasing concentrations of cDNA from an IgEsecreting myeloma cell line AF-10 (17) were subjected to PCR1' (Fig. $2 A$ ) and PCR2' (Fig. $2 B$ ). After the densitometry analysis, the intensity of each band was multiplied by the correction factor relating to its amplification rate. In both PCR strategies, the ratio between different isoforms remained constant regardless of the starting concentration of cDNA used. This demonstrated that after correcting for amplification rates no isoform was amplified preferentially.

\section{Results}

DEP enhances nasal IgE levels. The IgE levels in the nasal washes of 11 subjects taken $4 \mathrm{~d}$ after nasal challenge with DEP are shown in Fig. 3. IgE levels obtained after a total of 0.15 mg DEP challenge were not statistically significant $(P>0.05$, paired $t$ test) when compared with the same subjects challenged with saline alone. However, when the subjects were challenged with a total of $0.3 \mathrm{mg}$ of DEP $(0.15 \mathrm{mg} /$ nostril $)$, IgE levels were significantly higher compared with challenge with saline alone $(P<0.01$, paired $t$ test $)$. The ability to enhance IgE responses was not seen with $1 \mathrm{mg}$ of DEP. Indeed, when subjects were challenged with $1 \mathrm{mg}$ of DEP, it had an inhibitory effect as IgE levels were significantly lower $(P<0.01$, paired $t$ test) than after saline challenge.

Challenge with $0.3 \mathrm{mg}$ DEP induced an increase of IgE production at day 4 in all 11 subjects tested (range 1.4- to 10.2fold increase over prechallenge levels with a mean increase of $5.62 \pm 2.9$-fold for all 11 subjects). No IgE enhancement was observed after saline challenge. Fig. $4 \mathrm{~A}$ shows the data from four representative patients whose levels of $\operatorname{IgE}$ enhancement were intermediate. This increase in IgE (normalized for the levels of albumin) after challenge with $0.3 \mathrm{mg}$ of DEP was observed consistently at day 4 but was not present in lavage fluids at day 1 (data not shown). The nasal lavage IgE levels had returned to baseline by day 7 and remained there at day 10 (Fig. $4 \mathrm{~A}$ ). To ensure that there was no carry over effect between challenges, there was a period of at least $18 \mathrm{~d}$ between challenges. The baseline levels of nasal lavage IgE (normalized for the levels of albumin ) were monitored and seen to be similar before each challenge.

We extended our findings to determine if DEP enhanced IgE specifically or whether it affected the levels of multiple isotypes. We measured total IgA, IgE, IgG, and IgM levels in the same nasal washes of the same 11 subjects immediately 

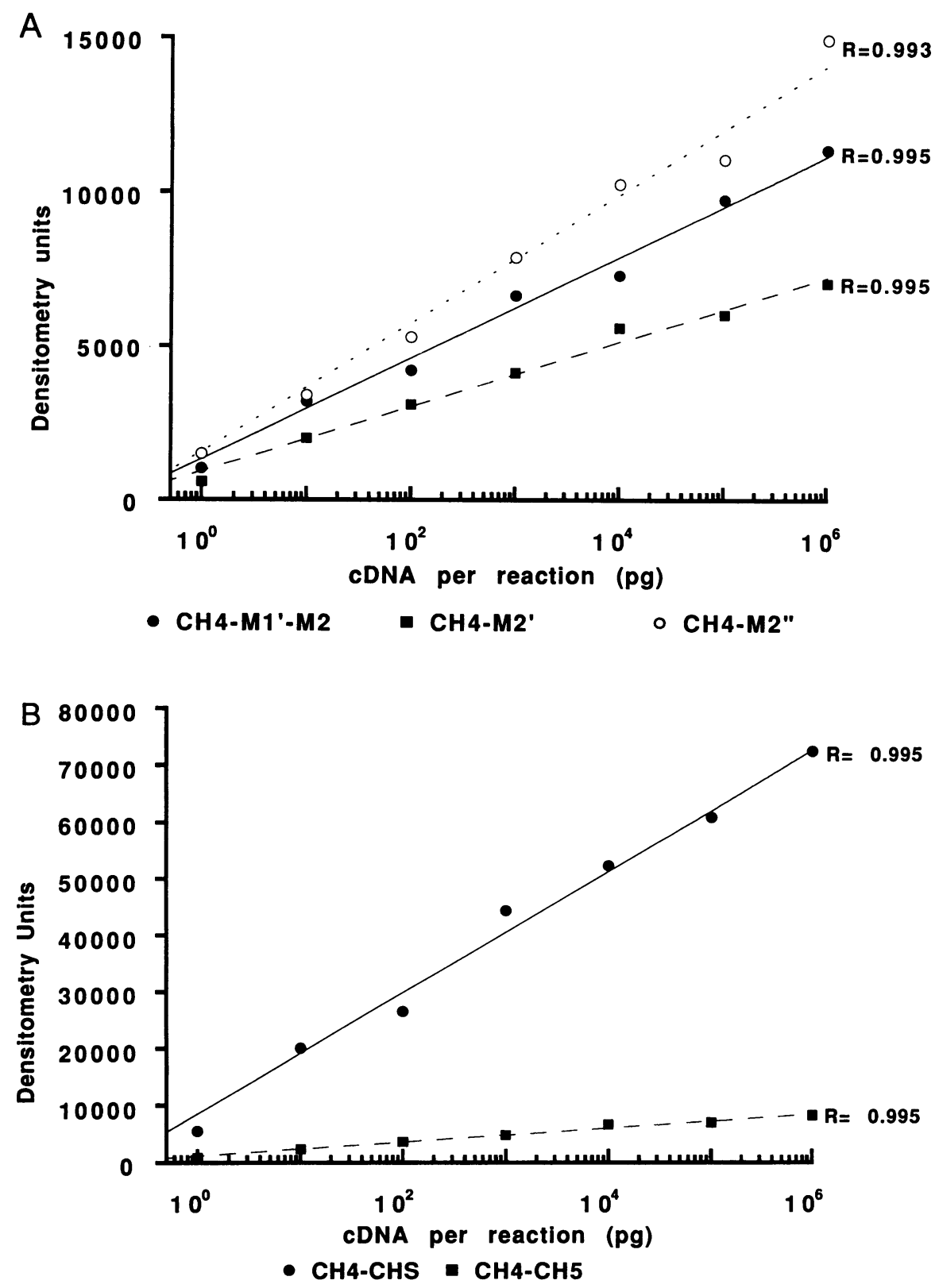

Figure 2. Verification of PCR strategy for the quantitation of the epsilon mRNA products generated. Increasing concentrations of cDNA from an IgE-secreting myeloma cell line AF-10 were subjected to PCR1 ' $(A)$ and PCR2' $(B)$. The amount of each isoform was quantitated for each concentration as explained in Methods. Linear regression was used to determine that there is a log-linear correlation between the starting concentration of the cDNA and the PCR yield and that the PCR strategies do not preferentially amplify any isoform.

before and 4, 7, and $10 \mathrm{~d}$ after challenge with a total of $0.3 \mathrm{mg}$ DEP. $\mathrm{IgG}_{4}$ levels were also measured, as expression of this subclass has often been linked with $\operatorname{IgE}$ production $(23,24)$. As serum levels of $\mathrm{IgG}_{3}$ are similar to that of $\mathrm{IgG}_{4}$ and $\mathrm{IgG}_{3}$ does not seem to be involved in the allergic response, the levels of this subclass in the nasal washes were also monitored. Albumin levels in the nasal lavage fluids were determined as it serves as a control for increased protein leakage.

The same four representative subjects are shown in regard to the various Ig isotypes in Fig. $4, A-G$. These subjects had intermediate IgE responses to DEP, and similar results were obtained for all 11 subjects studied. There was no significant elevation of albumin levels (Fig. $4 \mathrm{~B}$ ) over baseline values (mean 13,590 $\pm 1,987 \mathrm{ng} / \mathrm{ml}, n=11$ ) after DEP challenge either at day $4($ mean $16,180 \pm 2,511 \mathrm{ng} / \mathrm{ml})$ or any time $(P>0.05$, paired $t$ test, $n=11$ for all times). Similarly, after challenge with DEP, no consistent change over baseline values was observed in levels of total IgG (Fig. $4 C$ ), IgA (Fig. $4 D$ ), or
IgM (Fig. $4 E)(P>0.05$, paired $t$ test, $n=11$ for each isotype for each time). Interestingly, a rise in $\mathrm{IgG}_{4}$ levels was observed (Fig. $4 F$ ) in patients compared with their baseline levels. However, in contrast to nasal IgE levels that reached a maximum 4 $\mathrm{d}$ after challenge, in 8 out of the 11 subjects the increase in $\mathrm{IgG}_{4}$ levels was most pronounced after $7 \mathrm{~d}$ (mean $48.8 \pm 18.1$ $\mathrm{ng} / \mathrm{ml}, n=11)$ at which time $\mathrm{IgG}_{4}$ levels were significantly higher $(P<0.01$, paired $t$ test, $n=11)$ than prechallenge levels (mean $8.73 \pm 11.5 \mathrm{ng} / \mathrm{ml}, n=11$ ). The mean increase in $\mathrm{IgG}_{4}$ for the entire group of 11 subjects after $7 \mathrm{~d}$ was $23.2 \pm 3.4$-fold. By day $10, \mathrm{IgG}_{4}$ levels were no longer statistically enhanced $(P>0.05$, paired $t$ test, $n=11)$. In contrast, $\mathrm{IgG}_{3}$ levels (Fig. $4 G)$ did not increase at any time after DEP challenge $(P$ $>0.05$, paired $t$ test, $n=11$ ).

No increase in any Ig isotype including $\mathrm{IgG}_{4}$ was observed after challenge with 0.1 or $1 \mathrm{mg}$ DEP (data not shown). No significant change in the serum levels of $\operatorname{IgE}$ was detected in any patient at any time after DEP or saline challenge (data not 


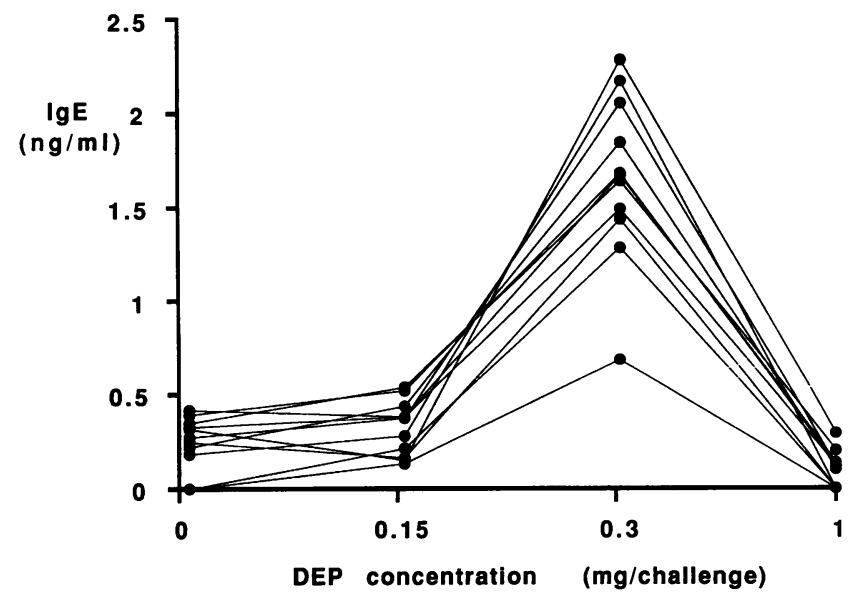

Figure 3. DEP induction of an IgE in the nose. 11 subjects were challenged in the nose with saline or a total of $0.15,0.3$, and $1 \mathrm{mg}$ of DEP. Each point represents the mean IgE level of four washes from each subject performed $4 \mathrm{~d}$ after challenge. IgE levels were determined by ELISA.

shown). There was no difference between the normal subjects and the asymptomatic atopic subjects in either resting IgE levels or in the response to DEP for any isotype.

DEP induces an increase in IgE- but not IgA-secreting cells. Cells recovered from nasal washes were used in a filter spotELISA (see Methods) to determine the number of Ig-secreting cells present before and after challenge with $0.3 \mathrm{mg}$ DEP compared with saline controls. After DEP challenge, the number of cells producing IgE detected in nasal washes increased to levels of $>1$ in 100,000 of the total cells recovered (Table I) compared with $<1$ in 2,000,000 of cells in lavages performed before challenge or after saline challenge. To assess whether this increase was due to non-isotype-specific effect such as infiltration of cells into the challenged and lavaged mucosa, the number of $\operatorname{IgA}$-secreting cells was determined in lavage fluids before and after DEP challenge using a similar filter spot-ELISA. Again, the number of spots detected was compared with a standard curve constructed using an appropriate IgA-secreting myeloma cell line. No change was observed in IgA-secreting cell density before or after challenge with either saline or DEP (Table II).

Epsilon mRNA isoform ratios are altered by DEP. In addition to the quantitative changes in IgE production noted above, we also assessed the ability of DEP to locally alter the qualitative IgE response by measuring the levels of five epsilon mRNAs generated by alternative splicing. Four of these epsilon mRNAs encode secreted IgE proteins that may well have differing biologic activities while the fifth epsilon mRNA codes for the large dominant epsilon membrane form of $\operatorname{IgE}(18,19)$. The baseline percentages for the isoforms were: CH4-M1 '-M2, 2.4\%; CH4-M2', 5.2\%; CH4-M2", 16.8\%; CH4-S, 67.5\%; $\mathrm{CH}^{\prime}{ }^{\prime}-\mathrm{CH} 5,8.0 \%$.

As shown in Fig. 5, all five isoforms of epsilon mRNA could be detected in cells recovered from washes performed before and $4 \mathrm{~d}$ after challenge with $0.3 \mathrm{mg}$ DEP. However, a change in the production of these epsilon mRNA isoforms was evident, not only was there an increase in the amount of mRNA present, the relative ratios between the different epsilon mRNAs were altered.

The total amount of epsilon mRNA obtained after DEP challenge increased nearly 28 -fold compared with prechallenge levels (Fig. 6). However, in cells from the eight subjects studied, only four of the five isoforms (CH4-M1'-M2, CH4-M2', CH4-M2", and CH4-S) increased by this relatively large amount (22-33-fold). The other isoform, the $\mathrm{CH} 4{ }^{\prime}-\mathrm{CH} 5$ splice variant that uses a unique splice acceptor and donor site and codes for a secreted isoform, increased considerably less (mean $3.4 \pm 1.8$-fold, $n=8$ ). Accordingly, the overall pattern of epsilon mRNAs changed with the ratio of $\mathrm{CH} 4-\mathrm{S}$ to $\mathrm{CH} 4$ '- $\mathrm{CH} 5$, for example, increasing from a ratio of 8.4:1 to a ratio of 80.9:1 (Fig. 6). There was no increase in the production of any isoform after challenge with saline (data not shown).

\section{Discussion}

In this report, we provide direct evidence that DEP can act in vivo to potentiate IgE production at the human respiratory mucosal surface. We show this quantitative increase in IgE occurs by expansion of the local population of IgE-secreting cells and is accompanied by a qualitative change in the pattern of epsilon mRNA isoforms produced. For our study, we simulated exposure by challenging volunteers with low doses of DEP delivered to a localized area. Challenge with a dose of DEP $(0.3 \mathrm{mg})$, approximately equivalent to breathing the outdoor air in Los Angeles for a 24-h period on an average day, caused a 1.4- to 10.2-fold increase in the amount of IgE found in nasal washes. This data shows that even relatively low level exposure to DEP may result in increased expression of IgE and thereby increase the likelihood of respiratory allergic disease.

Epidemiological studies have correlated the incidence of allergic airway disease with air quality over time or have compared it in diverse areas with different levels of pollution (1$4,25,26)$. These studies have provided indirect evidence of an increased incidence of asthma and atopy linked to airborne pollution. An important contributor to such airborne pollution is diesel exhaust emissions (27-30). Even certain nonoccupational settings such as passing a bus may result in acute exposure nearly 100 times higher than the recommended peak concentrations of PAH-DEP. In our study, for practical reasons we used a single bolus of DEP. However differences between sudden single exposure such as we used versus chronic low dose exposure will need to be elucidated.

Several animal studies have linked DEP and other environmental pollutants (as opposed to allergens) to atopy and bronchial hyperreactivity. Guinea pigs exposed to sulfur dioxide can develop allergic respiratory disorders (31) and increased antibody responses to allergens (32). Intranasal exposure of mice to DEP increases IgE antibody but not the IgG response to ovalbumin $(8,9)$. Similarly, rats and human exposed to tobacco smoke, which contains PAH such as benzo(a)pyrene also found in DEP, have increased serum IgE (33).

Here, we show that in DEP-challenged subjects there was an increase in the number of IgE-secreting cells from $<1$ in $2,000,000$ before challenge to $>1$ in $100,0004 \mathrm{~d}$ after challenge. There was no corresponding increase in $\operatorname{IgA}$-secreting cells, suggesting that DEP's activity by nasal inhalation may well show some selectivity for cells committed to IgE. This selectivity may also extend to $\mathrm{IgG}_{4}$ since DEP challenge did cause an increase in $\mathrm{IgG}_{4}$ protein production. $\mathrm{IgG}_{4}$ is associated with IgE production in a number of experimental systems (23, 24). The failure to see an increase in IgA (or IgM, total IgG, or $\mathrm{IgG}_{3}$ ) in nasal lavages after DEP challenge supports the view that DEP may act in an isotype-specific manner. However, the 

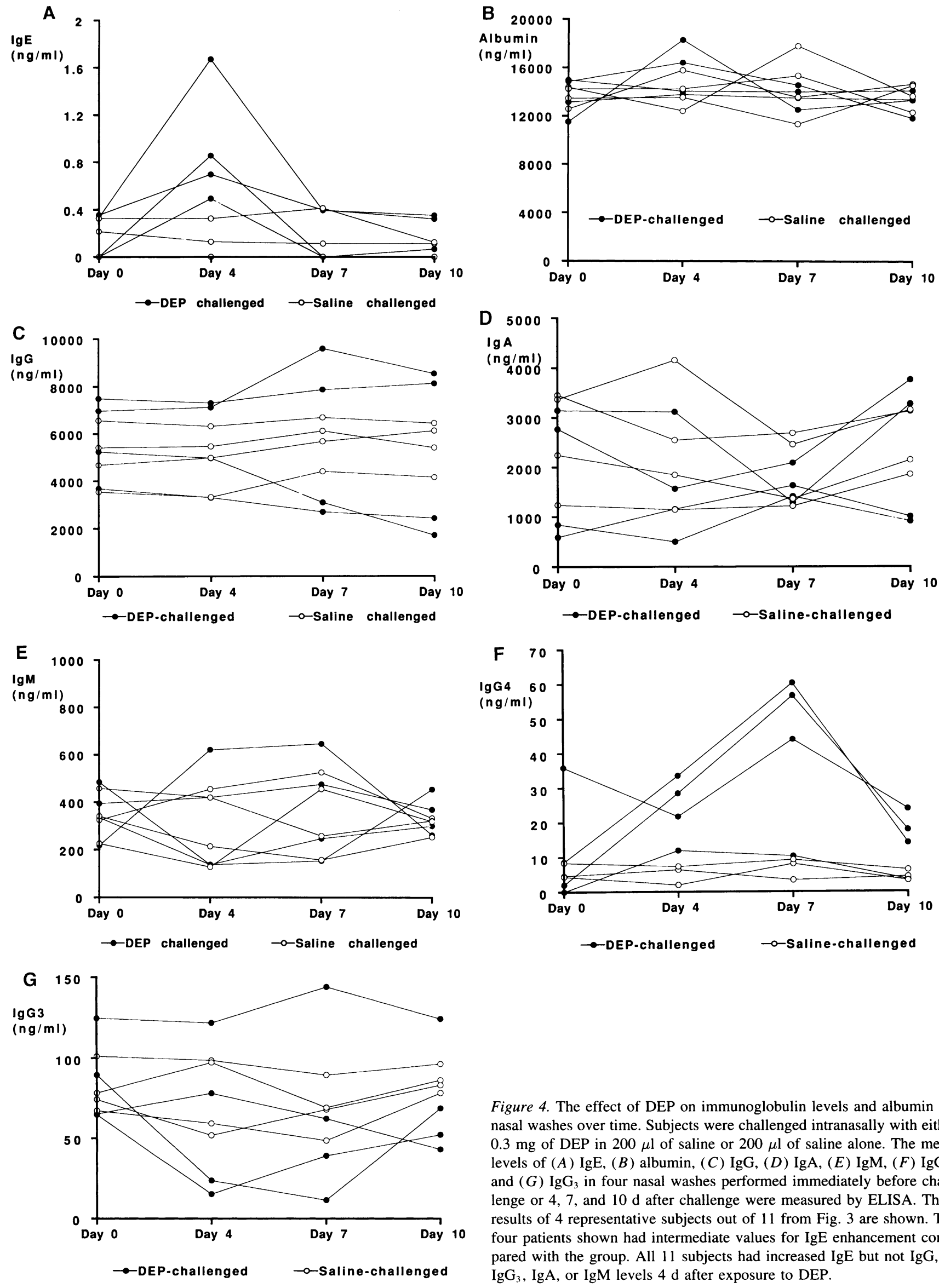

Figure 4. The effect of DEP on immunoglobulin levels and albumin in nasal washes over time. Subjects were challenged intranasally with either $0.3 \mathrm{mg}$ of DEP in $200 \mu \mathrm{l}$ of saline or $200 \mu \mathrm{l}$ of saline alone. The mean levels of $(A) \operatorname{IgE},(B)$ albumin, $(C) \operatorname{IgG},(D) \operatorname{IgA},(E) \operatorname{IgM},(F) \operatorname{IgG} 4$, and $(G) \mathrm{IgG}_{3}$ in four nasal washes performed immediately before challenge or 4,7 , and $10 \mathrm{~d}$ after challenge were measured by ELISA. The results of 4 representative subjects out of 11 from Fig. 3 are shown. The four patients shown had intermediate values for $\mathrm{IgE}$ enhancement compared with the group. All 11 subjects had increased IgE but not IgG, $\mathrm{IgG}_{3}, \operatorname{IgA}$, or IgM levels $4 \mathrm{~d}$ after exposure to DEP. 
Table I. DEP Enhances the Production of IgE-secreting Cells

\begin{tabular}{ccccc}
\hline & \multicolumn{4}{c}{ Number of IgE-producing cells per $10^{6}$ nasal lavage cells* } \\
\cline { 2 - 5 } Subject & Pre-saline & Post-saline & Pre-DEP & Post-DEP \\
\hline 1 & $0.64 \pm 0.13$ & $0.31 \pm 0.20$ & $0.16 \pm 0.08$ & $11.6 \pm 2.3$ \\
2 & 0 & 0 & 0 & $5.8 \pm 1.2$ \\
3 & 0 & 0 & $0.31 \pm 0.11$ & $23.4 \pm 4.6$ \\
4 & N.D. & N.D. & 0 & $5.0 \pm 0.8$ \\
5 & 0 & $0.31 \pm 0.14$ & N.D. & N.D. \\
6 & $0.31 \pm 0.24$ & $0.64 \pm 0.24$ & N.D. & N.D. \\
7 & $0.16 \pm 0.11$ & $0.16 \pm 0.12$ & N.D. & N.D. \\
8 & N.D. & N.D. & 0 & $6.4 \pm 1.4$ \\
9 & N.D. & N.D. & $0.31 \pm 0.31$ & $15.8 \pm 2.1$ \\
10 & $0.16 \pm 0.11$ & 0 & N.D. & N.D. \\
11 & N.D. & N.D. & $0.16 \pm 0.16$ & $14.6 \pm 1.8$
\end{tabular}

* Total cells recovered from nasal lavages before or $4 \mathrm{~d}$ after challenge with $0.3 \mathrm{mg}$ DEP or saline assayed for IgE cells by filter spot assay. The spots were counted, and the number of IgE-secreting cells present in the population was calculated by comparison with a standard curve (see Methods). All results shown are the mean \pm 1 SD of IgE-secreting cells $/ 10^{6}$ cells for five replicate wells. N.D., not determined.

possibility that an increase in local production of $\mathrm{Ig}$ can only be detected against a low background such as $\mathrm{IgE}$ and $\mathrm{IgG}_{4}$ cannot be discounted. However, the fact that $\mathrm{IgG}_{3}$ levels, which are similar to $\mathrm{IgG}_{4}$ in the serum, did not increase after DEP challenge suggests this effect shows some specificity.

Whether these results are mediated via an effect of DEP on the induction of cytokines (e.g., IL-4 or gamma interferon) is presently under investigation. It is known that DEP can have direct effects both on T cells (10) as well as on B cells (11a, 34 ). Our findings were not the result of tissue injury leading to nonspecific vascular leakage as there was no elevation of albumin levels after DEP challenge nor an increase on Ig iso-

Table II. DEP Does Not Enhance the Production of IgA-secreting Cells

\begin{tabular}{ccccc}
\hline \multicolumn{5}{c}{ Number of IgA-producing cells per $10^{6}$ nasal lavage cells* } \\
\cline { 2 - 5 } Subject & Pre-saline & Post-saline & Pre-DEP & Post-DEP \\
\hline 1 & 0 & 0 & $0.38 \pm 0.38$ & $0.76 \pm 0.38$ \\
2 & $0.76 \pm 0.21$ & $1.14 \pm 0.82$ & $1.14 \pm 0.88$ & $1.52 \pm 0.83$ \\
3 & $0.76 \pm 0.19$ & $0.38 \pm 0.24$ & $1.52 \pm 0.68$ & $0.76 \pm 0.48$ \\
4 & N.D. & N.D. & 0 & 0 \\
5 & $0.38 \pm 0.28$ & 0 & N.D. & N.D. \\
6 & $1.14 \pm 0.81$ & $0.76 \pm 0.42$ & N.D. & N.D. \\
7 & $1.52 \pm 0.43$ & $1.14 \pm 0.66$ & N.D. & N.D. \\
8 & N.D. & N.D. & $0.76 \pm 0.41$ & $1.14 \pm 0.76$ \\
9 & N.D. & N.D. & $1.52 \pm 0.83$ & $1.80 \pm 1.2$ \\
10 & $1.80 \pm 0.86$ & $1.14 \pm 0.78$ & N.D. & N.D. \\
11 & N.D. & N.D. & $0.76 \pm 0.64$ & $0.38 \pm 0.23$
\end{tabular}

* Total cells recovered from nasal lavages before or $4 \mathrm{~d}$ after challenge with $0.3 \mathrm{mg}$ DEP or saline assayed for IgA cells by filter spot assay.

The spots were counted, and the number of IgA-secreting cells present in the population was calculated by comparison with a standard curve (see Methods). All results shown are the mean \pm 1 SD of IgA-secreting cells $/ 10^{6}$ cells for five replicate wells. N.D., not determined.
$\underline{A}$

\section{Day 0 Day 4}

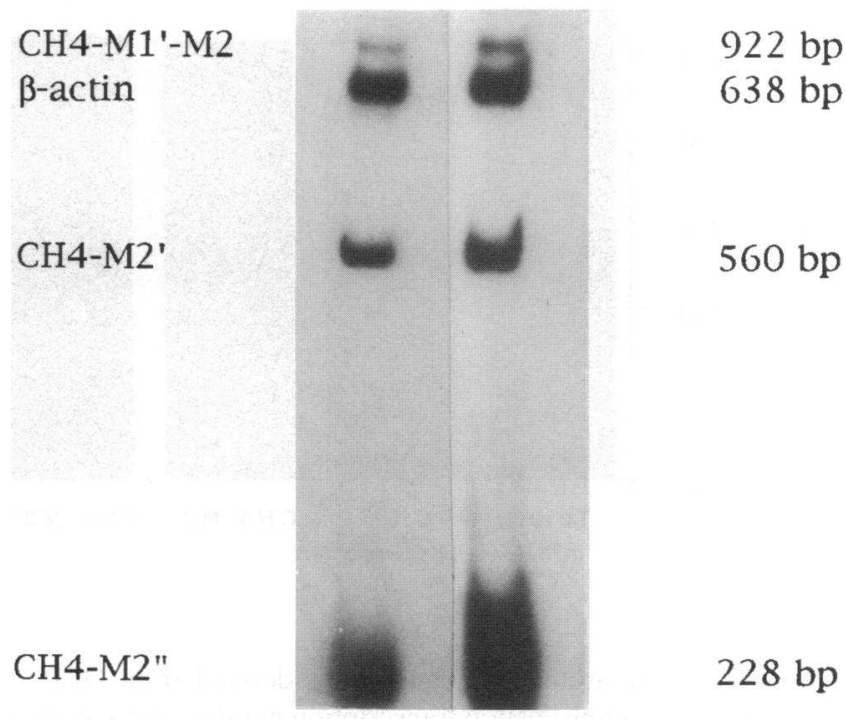

$\underline{B}$

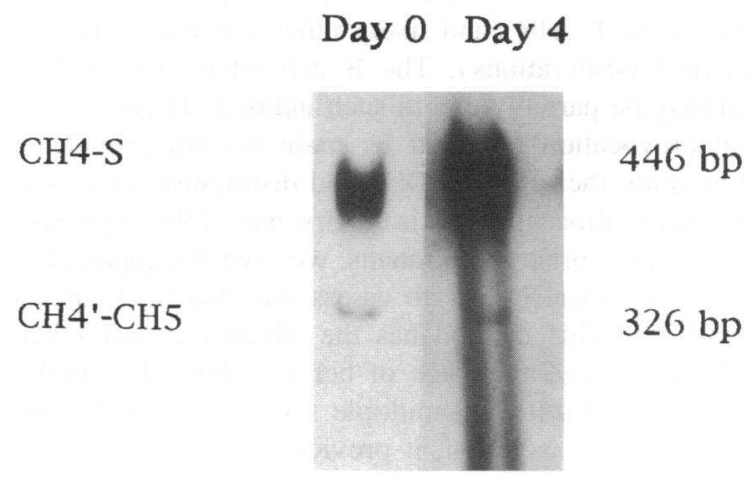

3-actin

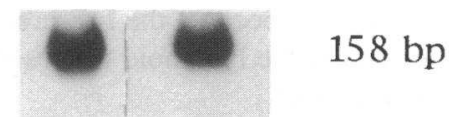

Figure 5. Autoradiographs of reverse transcriptase PCR products showing epsilon mRNA isoforms derived from RNA of cells recovered from nasal washes performed before and $4 \mathrm{~d}$ after challenge with $0.3 \mathrm{mg}$ of DEP. In $A$, the bands derived from PCR $1^{\prime}$ are shown. These represent the three epsilon mRNA isoforms CH4-M1 '-M2, CH4-M2', and CH4M2" and $\beta$-actin as labeled. In $B$, the bands derived from PCR2' are shown. These represent the two epsilon mRNA isoforms CH4-S and $\mathrm{CH} 4^{\prime}-\mathrm{CH} 5$ and $\beta$-actin as labeled. The size of the bands is shown on the right hand side. A substantial increase is apparent in all epsilon mRNAs except $\mathrm{CH} 4$ '-CH5. The results are from 1 representative subject, however, all 11 subjects responded in a similar way.

types other than IgE when adjusted for albumin levels. Furthermore, although the same number of cells (as determined by mRNA for the housekeeping gene $\beta$-actin) was used in our PCR assays, the amount of epsilon mRNA in cells from DEPexposed subjects markedly increased. We have established pre- 


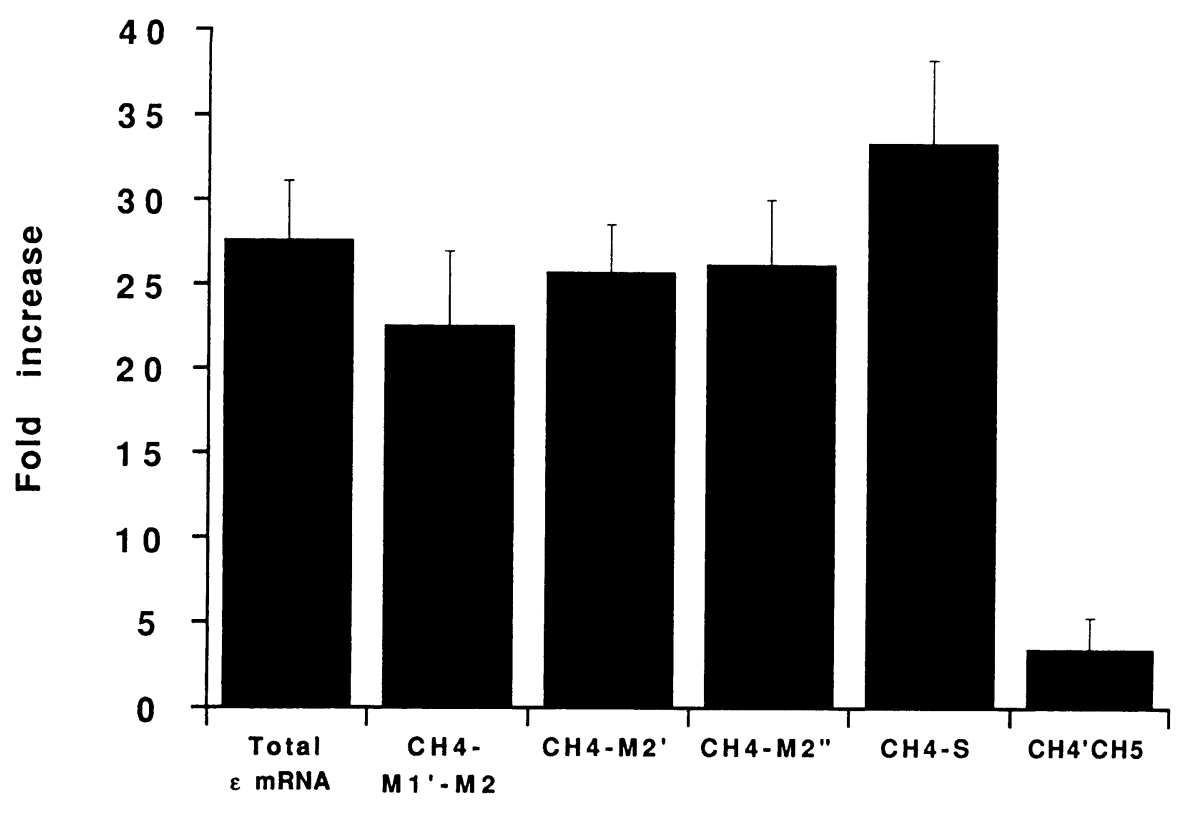

Figure 6. DEP alters the relative levels (pattern) of the epsilon mRNA isoforms. This figure shows the relative enhancement (as fold increase ) for each of the epsilon mRNAs noted as induced by DEP challenge $(0.3$ $\mathrm{mg}$ ). The amount of mRNA detected for each epsilon isoform from cells recovered in nasal washes performed before and $4 \mathrm{~d}$ after challenge with DEP was measured and standardized relative to the amount of $\beta$-actin present. The results shown are the mean and standard deviation of eight subjects. The total epsilon mRNA levels went up nearly 30 -fold as did mRNA for all of the splice variants except the $\mathrm{CH} 4{ }^{\prime}-\mathrm{CH} 5$ form. viously that the aromatic hydrocarbons derived from DEP do not induce germ line epsilon transcription nor does class switching $(11 a)$, it is unlikely that DEP acts by inducing B cells specifically into the IgE pathway. It is possible that DEP acts by expanding a preexisting population of IgE-committed memory B cells. However, we have now shown that DEP can also exert effects on $\mathrm{T}$ cells (and likely other cell types) in vivo (our personal observations). The B cell effects of DEP we observed may be partially due to such indirect effects.

Nasal provocation followed by nasal washes provided a method to study the effect of DEP and distinguish local from systemic effects. Since the nose is the first part of the respiratory tract to encounter airborne pollutants, we used the approach of others by using nasal lavage to assess the effects of inhaled pollutants (35). This method has the advantage over others such as bronchoalveolar lavage of being technically simpler, less traumatic, and allowing multiple samples to be obtained from one person. In addition, it provided us a model for the action of DEP on mucosal surfaces. The mucosal immune response is important because the IgE-producing cells are preferentially located in the lymphoid tissues that line the airways or gastrointestinal tract.

The change induced by in vivo DEP inhalation in the relative levels of the distinct epsilon mRNAs demonstrates a qualitative effect as well as a quantitative effect of IgE production. Alternate $3^{\prime}$ splicing results in the generation of multiple epsilon mRNA coding for distinct IgE isoforms from the single active epsilon gene in all human IgE-producing cells examined to date $(18,36,37)$. Features of the mRNAs and their protein products have been discussed elsewhere (18). Using quantitative PCR techniques, five of the main isoforms of epsilon mRNA were detected in fresh cells obtained from nasal washes. This is the first report of the detection of such epsilon mRNA isoforms in fresh normal nonmyeloma noncultured B cells as well as the first demonstration that the epsilon mRNA isoform pattern is not fixed in vivo.

The most abundant isoforms before DEP challenge were the classic secreted $(\mathrm{CH} 4-\mathrm{S})$ and a novel secreted epsilon form designated $\mathrm{CH} 4-\mathrm{M} 2$ " resulting from the splicing of $\mathrm{CH} 4$ to a distant $3^{\prime}$ splice acceptor site. After DEP challenge, there was a change in the overall pattern of epsilon isoform mRNAs observed. The $\mathrm{CH} 4-\mathrm{S}: \mathrm{CH} 4{ }^{\prime}-\mathrm{CH} 5$ was $8.4: 1$ before challenge, $4 \mathrm{~d}$ later it had increased to $80.9: 1$. The $\mathrm{CH} 4{ }^{\prime}-\mathrm{CH} 5$ mRNA uses a novel splice donor site in the middle of $\mathrm{CH} 4$ that is spliced to 107-bp cryptic $\mathrm{CH} 5$ exon immediately downstream of $\mathrm{CH} 4$. As such, $\mathrm{CH}^{\prime}{ }^{\prime}-\mathrm{CH} 5$ is the only isoform that uses the novel $5^{\prime}$ donor site and the $\mathrm{CH} 5$ acceptor site rather than the $\mathrm{CH} 4$ donor site with alternative acceptor sites. Whether DEP preferentially rejects the $\mathrm{CH} 4$ ' donor or $\mathrm{CH} 5$ acceptor sites or selects for the $\mathrm{CH} 4$ donor site is currently being investigated. In vitro, DEP and IL-10 have a similar effect while other stimuli can alter the splice pattern differently (our personal observation). This suggests that either the DEP effect is via cytokines or that DEP and these other stimuli use the same mechanism at the B cell level. Ongoing studies have shown that different disease states ( such as common variable or hyper IgE immunodeficiency B cells) have different epsilon splice patterns. Therefore, this change in isoform pattern may have a potential biological role.

It is likely that the IgE-related changes we observed ultimately result from complex interplay involving the effects of DEP and their chemical constituents on B cells, T cells, and their cytokines, as well as other cells such as monocytes/macrophages and even epithelial cells. For example, we have observed similar changes on both IgE levels and epsilon isoform patterns by IL-10 (our personal observation). Our results suggest that DEP can enhance human $B$ cell differentiation and that exposure to DEP or their constituents may augment human allergic disease responses by enhancing the production of $\operatorname{IgE}$ antibodies.

\section{Acknowledgments}

We thank Dr. Shigeru Takafuji, Department of Medicine and Physical Therapy, Faculty of Medicine, University of Tokyo, Tokyo, Japan for his kind gift of diesel exhaust particles.

This work was supported by U.S. Public Health Service grants AI15251 and AI-34567 (UCLA Asthma, Allergy and Immunologic Disease Center) and gifts from the Dorothy fund, and the Asthma Research Foundation. Dr. Diaz-Sanchez was the recipient of the McClure Foundation Fellowship from the Los Angeles Chapter of the Asthma and Allergy Foundation. 


\section{References}

1. Takafuji, S., S. Suzuki, M. Muranaka, and T. Miyamoto. 1989. Influence of environmental factors on IgE production. IgE, mast cells and the allergic response. Ciba Found. Symp. 147:188-204.

2. Corbo, G. M., F. Forastiere, V. Dell'Orco, R. Pistelli, N. Agabati, B. De Stefanis, G. Ciappi, and C. A. Perucci. 1993. Effects of environment on atopic status and respiratory disorders in children. J. Allergy Clin. Immunol. 92:616623

3. Bascom, R., T. Kulle, A. Kagey-Sobotka, and D. Proud. 1991. Upper respiratory tract environmental tobacco smoke sensitivity. Am. Rev. Respir. Dis. 143:1304-1311.

4. Emanuel, M. B. 1988. Hay fever, a post industrial revolution epidemic: a history of its growth during the 19th century. Clin. Allergy. 18:295-304.

5. Kaneko, S., K. Shimada, H. Noriuchi, A. Endo, M. Kodama, N. Shinoda, T. Imai, and K. Takiguchi. 1980. Nasal allergy and air pollution. Oto-RhinoLaryngol. Tokyo. 23:270-279.

6. Jensen, E. J., B. Pedersen, E. Schmidt, and R. Dahl. 1992. Serum IgE in nonatopic smokers, nonsmokers, and recent exsmokers: relation to lung function, airway symptoms, and atopic predisposition. J. Allergy Clin. Immunol. 90:224229.

7. Muranaka, M., S. Suzuki, K. Koizumi, S. Takafuji, T. Miyamoto, R. Ikemori, and H. Tokiwa. 1986. Adjuvant activity of diesel-exhaust particulates for the production of IgE antibody in mice. J. Allergy Clin. Immunol. 77:616-623.

8. Takafuji, S., S. Suzuki, K. Koizumi, K. Tadokoro, T. Miyamoto, R. Ikemori, and M. Muranaka. 1987. Diesel-exhaust particulates inoculated by the intranasal route have an adjuvant activity for IgE production in mice. J. Allergy Clin. Immunol. 79:639-645.

9. Takafuji, S., S. Suzuki, K. Koizumi, K. Tadokoro, H. Ohashi, M. Muranaka, and T. Miyamoto. 1989. Enhancing effect of suspended particulate matter on the IgE antibody production in mice. Int. Arch. Allergy Appl. Immunol. 90:1-7.

10. Nagarkatti, P. S., G. D. Sweeney, J. Gauldie, and D. A. Clark. 1984. Sensitivity to suppression of cytotoxic $T$ cell generation by $2,3,7,8$-tetrachlorodibenzo- $p$-dioxin (TCDD) is dependent on the Ah genotype of the murine host. Toxicol. Appl. Pharmacol. 72:169-176.

11. Morris, D. L., J. G. Karras, and M. P. Holsapple. 1993. Direct effects of 2, $3,7,8$-tetrachlorodibenzo- $p$-dioxin (TCDD) on responses to lipopolysaccharide (LPS) by isolated murine B-cells. Immunopharmacology. 26:105-112.

11a. Takenaka, H., K. Zhang, D. Diaz-Sanchez, A. Tsein, and A. Saxon. 1994. Enhanced human IgE results from exposure to the aromatic hydrocarbons in diesel exhaust: direct effects on B cell IgE production. J. Allergy Clin. Immunol. In press.

12. Macy, E., D. M. Kemeny, and A. Saxon. 1988. Enhanced ELISA: how to measure less than 10 picograms of a specific protein (immunoglobulin) in less than 8 hours. FASEB (Fed. Am. Soc. Exp. Biol.) J. 2:3003-3010.

13. Zhang, K., E. A. Clark, and A. Saxon. 1991. CD40 stimulation provides an IFN- $\gamma$-independent and IL-4-dependent differentiation signal directory to human B cells for IgE production. J. Immunol. 146:1836-1842.

14. King, C. L., G. Thyphronitis, and T. B. Nutman. 1990. Enumeration of IgE secreting B cells. A filter spot-ELISA. J. Immunol. Methods. 132:37-43.

15. King, C. L., R. W. Poindexter, J. Ragunathan, T. A. Fleisher, E. A. Ottesen, and T. B. Nutman. 1991. Frequency analysis of IgE-secreting B lymphocytes in persons with normal or elevated serum IgE levels. J. Immunol. 146:1478-1483.

16. Thyphronitis, G., E. E. Max, and F. D. Finkelman. 1991. Generation and cloning of stable human IgE-secreting cells that have rearranged the C $\epsilon$ gene. J. Immunol. 146:1496-1502.

17. Hassner, A., and A. Saxon. 1984. Isotype-specific suppressor T-cells for IgE synthesis activated by IgE-anti-IgE immune complexes. J. Immunol. 132:2844-2849.

18. Zhang, K., E. E. Max, H.-K. Cheah, and A. Saxon. 1994. Complex alterna- tive RNA splicing of $\epsilon$ immunoglobulin transcripts produces mRNAs encoding four potential secreted protein isoforms. J. Biol. Chem. 269:456-462.

19. Zhang, K., A. Saxon, and E. E. Max. 1992. Two unusual forms of human immunoglobulin E encoded by alternative RNA splicing of $\epsilon$ heavy chain membrane exons. J. Exp. Med. 176:233-243.

20. Go, A. S., J. A. Zack, S. J. Arrigo, and I. S. Y. Chen. 1990. Quantitative polymerase chain reaction. In Techniques in HIV Research. A. Aldonini and B. D. Walker, editors. Stockton Press, New York. 113-119.

21. Yamamura, M., X.-H. Wang, J. D. Ohmen, K. Uyemura, T. H. Rea, B. R. Bloom, and R. L. Modlin. 1992. Cytokine patterns of immunologically mediated tissue damage. J. Immunol. 149:1470-1475.

22. Staynov, D. Z., and T. H. Lee. 1992. Expression of interleukin-5 and granulocyte-macrophage colony stimulating factor in human peripheral blood mononuclear cells after activation with phorbal myristate acetate. Immunology. 75:196-201.

23. Jabara, H. H., S. M. Fu, R. S. Geha, and D. Vercelli. 1990. CD40 and IgE: synergism between anti-CD40 monoclonal antibody and interleukin 4 in the induction of IgE synthesis by highly purified human B cells. J. Exp. Med. 172:1861-1864.

24. Armitage, R. J., B. M. Macduff, M. K. Spriggs, and W. C. Fanslow. 1993. Human B cell proliferation and Ig secretion induced by recombinant CD40 ligand are modulated by soluble cytokines. J. Immunol. 150:3671-3680.

25. Turner, K. J. 1989. Epidemiology of the allergic response. In Mast Cells and the Allergic Response. C. Wiley, editor. Ciba Found. Symp. 147:205-229.

26. Ishizaki, T., K. Koizumi, R. Ikemori, Y. Ishiyama, and E. Kushibiki. 1987. Studies of prevalence of Japanese cedar pollinosis among the residents in densely cultivated area. Ann. Allergy. 58:265-270.

27. Rykowski, R. A., and A. J. Brochu. 1986. Diesel particulate emissions: the United States experience with extrapolations to Europe and Japan. In Aerosols. S. D. Lee, T. Schneider, L. D. Grant, and P. J. Verkerk, editors. Lewis Publishers, Chelsea, MI. 443-457.

28. Spangler, J. D., and J. M. Samet. 1991. A perspective on indoor and outdoor air pollution. In Indoor Air Pollution: A Health Perspective. J. M. Samet and J. D. Spangler, editors. Johns Hopkins University Press, Baltimore, MD. 132.

29. Wallace, L. A. 1991. Volatile organic compounds. In Indoor Air Pollution: A Health Perspective. J. M. Samet and J. D. Spangler, editors. Johns Hopkins University Press, Baltimore, MD. 252-272.

30. Ryan, P. B., and W. E. Lambert. 1991. Personal exposure to indoor air pollution. In Indoor Air Pollution: A Health Perspective. J. M. Samet and J. D. Spangler, editors. Johns Hopkins University Press, Baltimore, MD. 109-127.

31. Matsumara, Y. 1970. The effects of ozone, nitrogen dioxide, and sulfur dioxide on the experimentally induced allergic disorder in guinea pigs. Am. Rev. Respir. Dis. 102:430-437.

32. Riedel, F., M. Krämer, C. Scheibenbogen, and C. H. L. Rieger. 1988. Effects of $\mathrm{SO}_{2}$ exposure on allergic sensitization in the guinea pig. J. Allergy Clin. Immunol. 82:527-534.

33. Zetterström, O., S. L. Nordvall, B. Bjorksten, S. Ahlstedt, and M. Stelander. 1985. Increased IgE antibody responses in rats exposed to tobacco smoke. J. Allergy Clin. Immunol. 75:594-598.

34. Kramer, C. M., K. W. Johnson, R. K. Dooley, and M. P. Holsapple. 1987. $2,3,7,8$-Tetrachlorodibenzo- $p$-dioxin (TCDD) enhances antibody production and protein kinase activity in murine B cells. Biochem. Biophys. Res. Commun. 145:25-33.

35. Koren, H. S., G. E. Hatch, and D. E. Graham. 1990. Nasal lavage as a tool in assessing acute inflammation in response to inhaled pollutants. Toxicology. 60:15-25.

36. Peng, C., F. M. Davis, L. K. Sun, R. S. Liou, Y. Kim, and T. W. Chang 1992. A new isoform of human membrane bound IgE. J. Immunol. 148:129136.

37. Hellman, L. 1993. Characterization of four novel e chain mRNA and a comparative analysis of genes for immunoglobulin $\mathrm{E}$ in rodents and man. Eur. J. Immunol. 23:159-167. 